\title{
Monitoreo con SIG de la calidad de la atmósfera urbana para la gobernanza local: el caso de Madrid
}

\author{
Rosa Cañada Torrecilla y \\ Antonio Moreno Jiménez
}

\section{Resumen}

Las políticas locales están demandando estudios de evaluación periódicos para determinar si la evolución urbana es positiva o negativa, desde diferentes principios o perspectivas como el de los desequilibrios espaciales. Tal es el caso de Madrid, donde anualmente se realiza un examen de los mismos, incluyendo los aspectos de polución atmosférica, dada su repercusión en facetas como la salud ciudadana, la sostenibilidad, la justicia ambiental, etc.

En esta contribución se presenta una actividad de asesoramiento municipal en esa línea desde la universidad, que implica análisis abundantes basados en geotecnologías. Como ilustración se describe someramente la metodología y los resultados para valorar las desigualdades intraurbanas en tres contaminantes importantes (NO2, ozono y PM10). Para ello se aplican técnicas de interpolación espacial a datos registrados en las estaciones para estimar la polución en el área urbana; los resultados luego se sintetizan por distritos municipales, base para la comparación y la toma de decisiones.

Palabras clave: desequilibrios urbanos / polución atmosférica / interpolación espacial / gobernanza urbana / Madrid.

\section{Abstract}

Local policies are requesting periodical assessments to elucidate whether urban evolution is positive or negative, according to different principles or points of view, such as the spatial disequilibria one. This is the case of Madrid, where on a yearly basis it is performed an exam of them, including issues such as atmospheric pollution, provided that it affects to human health, sustainability, environmental equity, etc.

In this contribution it is exposed a consulting activity made from the university, involving lots of geotechnologies based analysis. As an example, it is described here the 
methods and main results to assess the intraurban inequalities in outstanding air pollutants (NO2, ozone and PM10). To this end, using data registered in sampling stations, spatial interpolation is applied to estimate the pollution for the whole urban area; the results are then summarized for municipal districts, as a basis for comparison and decision taking.

Keywords: urban disequilibria / atmospheric pollution / spatial interpolation / urban governance / Madrid.

Rosa Cañada Torrecilla (rosa.canada@uam.es); Antonio Moreno Jiménez (antonio.moreno@uam.es). Departamento de Geografía, Universidad Autónoma de Madrid, España. Este trabajo se ha elaborado parcialmente en el marco del proyecto de investigación "Contaminación atmosférica urbana y justicia ambiental: metodología de evaluación y estudio de casos con sistemas de información geográfica", financiado por el Ministerio de Ciencia e Innovación de España, referencia CSO2011-26177. 


\section{INTRODUCCIÓN}

El contexto de actual gobernanza local aparece caracterizado por algunos rasgos singulares que conviene recordar: información sobre las ciudades más abundante y de mejor calidad, mayores posibilidades de compartirla entre los agentes interesados (stakeholders) a través de las redes de comunicaciones, superior sensibilidad ciudadana hacia los problemas del municipio, disponibilidad de cauces más eficaces para la participación ciudadana en los procesos de formación de decisiones públicas, crecientes relaciones, bien de competencia, bien de cooperación, entre ciudades (en línea con los postulados del marketing territorial), requerimientos más intensos desde organismos internacionales (e. g. la UE) para aplicar protocolos de evaluación de distintas facetas de las ciudades, de difusión de los resultados obtenidos y de diseño y puesta en marcha de políticas y acciones que mejoren la realidad presente (e. g. medidas correctivas o de impulso), etc. Todo ello está haciendo brotar unas necesidades de seguimiento periódico del estado y evolución urbanos en aspectos como por ejemplo la calidad de vida, la situación social, el desenvolvimiento de las actividades económicas, el medio ambiente, etc.

En consecuencia, cada vez más los gobiernos locales están asumiendo que, de cara a avanzar por la senda del desarrollo, no solo las fórmulas a medio y largo plazo, como el planeamiento urbano tradicional o los planes estratégicos, sino también los planes sectoriales y las actuaciones de menor alcance y la gestión urbana deben entenderse, concretarse y evaluarse desde principios importantes socialmente compartidos. La asunción de tales principios, sobre los que remitimos a otros trabajos (vid. Moreno y Vinuesa, 2009), implica una permanente labor de análisis sobre las diversas facetas de la ciudad, de cara a disponer de informes fundados sobre el estado y evolución de las mismas, a establecer la convergencia-divergencia de la ciudad respecto a los principios del desarrollo, a orientar decisiones mejor, a evaluar ex post los logros de políticas y actuaciones, etc. Sobre tales premisas cobra sentido la contribución que desde la Geografía cabe realizar, por su acreditada labor de estudio sobre las ciudades, desde la complementariedad de las perspectivas sectorial e integral u holista.

Esa capacidad de la disciplina ha sido fortalecida e impulsada sobre todo desde el advenimiento de las geotecnologías, las cuales están haciendo posible desarrollar una "inteligencia del territorio basada en TIG", que ofrece como resultado "conocimiento espacial" útil de manera ágil, rigurosa y asequible para los agentes y stakeholders urbanos. Como una materialización de esta sugerente línea de 
aplicación de las TIG, en esta contribución se describe una experiencia actual, desarrollada mediante una colaboración del Departamento de Geografía de la Universidad Autónoma de Madrid con el Observatorio Económico del Ayuntamiento de Madrid, en la que se aborda periódicamente el examen y valoración de los desequilibrios y reequilibrios en dicha ciudad.

En el apartado siguiente se describe someramente la actividad desarrollada, para exponer después un ejemplo de los datos tratados, los análisis ejecutados y los resultados obtenidos, concretamente el concerniente a la calidad del ambiente atmosférico. Tras ello se realiza un balance y conclusiones acerca de esta ilustrativa experiencia de análisis geográfico urbano orientado a la gobernanza local.

\section{LA ACTIVIDAD DE MONITOREO DE LOS EQUILIBRIOS Y REEQUILIBRIOS INTRAURBANOS DE LACIUDAD DE MADRID}

Como en otro lugar hemos señalado (Moreno y Vinuesa, 2009), el concepto de desequilibrio alude a desigualdades y desajustes territoriales negativamente connotados, los cuales deben distinguirse del término más general y "aséptico" de diferencias espaciales, que simplemente revela contrastes, sin juicio de valor explícito, y que por ende podrían resultar bien positivos y valiosos, bien negativos. Los desequilibrios son la consecuencia de dinámicas muy heterogéneas en las que concurren factores varios - unos de índole físico-natural, otros de origen humano -. Como resultado de ellas se conforman estructuras espaciales que poseen unas implicaciones (negativas o positivas), a veces importantes. Ciertos procesos, en su decurso, ocasionan la diferenciación espacial, en tanto que otros pueden mostrar una mayor propensión a reducir las desigualdades y a uniformar el territorio. Cuando tales desigualdades comportan consecuencias indeseables o graves, se habla de desequilibrios y brota la necesidad de diagnosticarlas, prevenirlas o corregirlas mediante actuaciones (e.g. políticas, planes, etc.).

El gobierno municipal de la ciudad de Madrid ha manifestado desde hace varios años una preocupación acerca de la situación y evolución de los desequilibrios intraurbanos a través del Observatorio Económico (http://www.esmadrid.com/es/ observatorio-economico), una unidad de análisis especializada en la elaboración e impulso de estudios sobre la realidad económica y territorial del municipio. En ese contexto, y junto con especialistas de otros campos y entidades, desde mediados de la pasada década un equipo de investigación de la Universidad Autónoma de Madrid ha podido colaborar en el análisis de los desequilibrios-reequilibrios de Madrid. La voluntad inicial estribaba en disponer de evaluaciones periódicas de 
los mismos, considerando un espectro muy amplio de facetas urbanas, lo que dio lugar a informes extensos basados en más de cien indicadores (vid. Cuadrado et al., 2005; Moreno y Vinuesa, 2009). En fechas recientes y por diversas razones, los estudios se han redimensionado dando lugar a documentos más compactos, aunque manteniendo su vocación de abordar una gama básica de dimensiones de la ciudad (vid. Moreno et al., 2011 y 2012).

El destino primero y, por ende, las utilidades inmediatas de este seguimiento anual de la ciudad estriban en que sus resultados sean conocidos y manejados por la Dirección General de Participación Ciudadana, órgano municipal responsable de encauzar las relaciones con las organizaciones vecinales de cara a la formación de los "planes de barrio". Éstos son instrumentos de intervención integral en partes selectas de la ciudad, que resultan de la negociación entre Ayuntamiento y representantes ciudadanos. Los análisis realizados por nuestro equipo buscan dar una visión sintética acerca de si globalmente tales planes (que son además individualmente evaluados) generan cambios positivos, es decir, una reducción de las desigualdades en el conjunto de la ciudad. A tal fin los informes son puestos simultánea y gratuitamente a disposición de políticos, expertos y ciudadanos en general a través de una publicación periódica (Barómetro de Economía de la Ciudad de Madrid'). Se trata por tanto de una expresión genuina de la sociedad del conocimiento, con la que se persigue ayudar a la evaluación de decisiones locales que palíen los desequilibrios intraurbanos detectados en diversas facetas urbanas (e.g. en la calidad del aire urbano).

Los informes realizados en la actualidad adoptan como unidad espacial los distritos municipales, por cuanto conforman divisiones administrativas territoriales relevantes para la formación de decisiones, por lo que el diagnóstico valorativo ha de atenerse a esa escala. Ello no obsta para que, en el curso del análisis, se elaboren y obtengan resultados con una mayor desagregación espacial, si bien finalmente han de sintetizarse y presentarse a ese nivel.

Las facetas tratadas en los estudios más recientes conciernen a la composición de la población, nivel socioeconómico, vivienda, actividad económica, transporte y accesibilidad, y medio ambiente. Para cada una de ellas se obtiene un conjunto de indicadores representativos (alrededor de veinte), los cuales son cartográficas y gráficas. A través de los resultados se trata de desvelar en qué

${ }^{1}$ Descarga en el portal: http://www.esmadrid.com/es/portal.do?IDM=137\&NM=3\&TR=C\&IDR=2032 
medida las desigualdades entre los distritos pueden ser interpretadas en términos de desequilibrios intraurbanos que incidan sobre varios principios de evaluación importantes: la calidad de vida ciudadana, la competitividad urbana, la sostenibilidad, la cohesión, la equidad territorial y la justicia ambiental. En el aspecto ambiental concretamente se examinan los niveles medios anuales de concentración de contaminantes en la atmósfera urbana (dióxido de nitrógeno, ozono y $\mathrm{PM}_{10}$ ), cuyo tratamiento se describe más adelante.

Pues bien, desde la óptica de la elaboración técnica de los mencionados informes, resulta procedente señalar aquí que, aparte de una importante labor de recopilación de datos, en la que la colaboración de los propios servicios del Ayuntamiento resulta sobresaliente, el tratamiento y análisis de la información ha de basarse y de manera muy intensa en los SIG y diversas técnicas de análisis estadístico-espacial. Para ciertos indicadores, como es el caso de la valoración de las desigualdades intraurbanas en la contaminación atmosférica, el proceso reviste un interés, a nuestro juicio, que justifica su presentación aquí como una ilustración de las potencialidades que las TIG proporcionan para el asesoramiento regular a los gobiernos locales de forma asequible y sistemática. A ello se dedican los apartados siguientes.

\section{LA EVALUACIÓN DE LAS CONDICIONES AMBIENTALES: LA CALIDAD DEL AIRE}

Desde el punto de vista de los desequilibrios espaciales, el examen periódico de la contaminación atmosférica urbana resulta pertinente por cuanto ésta incide en varios de los principios anteriormente reseñados como relevante; en particular, la sostenibilidad, la calidad de vida y el bienestar humano, junto con la justicia ambiental, están directamente condicionados por esa calidad del aire. Las diferencias intraurbanas pueden ocasionar además serias penalizaciones a ciertas áreas y subpoblaciones de la ciudad que deben desvelarse. De otra manera, la mayor 0 menor polución está también asociada a la desigual eficiencia con que funcionan ciertas actividades dentro del espacio urbano (por ejemplo, el transporte o los sistemas de calefacción).

Ahora bien, la valoración de la contaminación atmosférica urbana mayoritariamente se basa en los datos muestrales registrados por las estaciones de una red de calidad del aire que deja amplios espacios sin cubrir y no permiten una apreciación de las condiciones ambientales del conjunto de la ciudad. Para informar con 
mejor fundamento a los responsables del gobierno local, se precisa abordar primero una estimación de la contaminación en todo el espacio poblado del municipio, para elaborar después una síntesis por unidades administrativas territoriales (e.g. los distritos municipales), que facilite la apreciación de los logros globales de las actuaciones municipales que inciden en el medio ambiente urbano.

Los contaminantes atmosféricos más significativos para analizar y evaluar la calidad del aire que se están utilizando son el dióxido de nitrógeno $\left(\mathrm{NO}_{2}\right)$, el ozono $\left(\mathrm{O}_{3}\right)$ y las partículas en suspensión inferiores a $10 \mu\left(\mathrm{PM}_{10}\right)$ correspondientes al año 2011; otros examinados con anterioridad, como el $\mathrm{SO}_{2}$, no resultan en estos momentos preocupantes. Pues bien, esos tres contaminantes experimentaron problemas en ese año para cumplir los objetivos fijados por la normativa vigente, ya que presentaron superaciones de los valores límite horario y anual de protección a la salud humana. El objetivo de las políticas de los países es conseguir un medio ambiente en el que el nivel de contaminación no dé lugar a efectos perjudiciales para la salud humana y el medio ambiente, y en el que los grupos de población más vulnerables estén protegidos.

Actualmente se están desarrollando políticas a nivel europeo entre las que destacan el Sexto Programa de Acción en Materia de Medio Ambiente (6PAMA), la Estrategia sobre Medio Ambiente y Salud, el Plan de Acción 2004-2010 de la UE, el proceso paneuropeo de Medio Ambiente y Salud de la Organización Mundial de la Salud (OMS) y la Directiva 2008/50/CE relativa a la calidad del aire ambiente y a una atmósfera más limpia en Europa, que es la norma europea más reciente que regula la evaluación de la calidad del aire y establece los niveles de los contaminantes que son más relevantes por sus efectos en la salud humana, que son precisamente los tres que se estudian en este trabajo (véase tabla 1). Directiva Europea incorporada a nuestra legislación mediante el Real Decreto 102/2011 del 28 de enero.

Tabla 1. Valores límites de la calidad del aire

\begin{tabular}{|c|c|c|c|}
\hline CONTAMINANTES & VALOR LFMITE DLARIO & $\begin{array}{l}\text { VAIOR } \\
\text { LMMTIE } \\
\text { ANUAL }\end{array}$ & $\begin{array}{l}\text { VALOR OBVETIVO } \\
\text { ANO 2010-2012 }\end{array}$ \\
\hline $\begin{array}{l}\text { Dióxido de nitrógeno } \\
\text { NO, }\end{array}$ & $\begin{array}{l}200 \mu \mathrm{g} / \mathrm{m}^{3} \text { no podrá superarse en más de } \\
18 \text { ocasiones al año }\end{array}$ & $40 \mu g / \mathrm{m}^{3}$ & \\
\hline Ozono $\mathrm{O}_{3}$ & $\begin{array}{l}\text { Umbral de información a la población: } \\
180 \mu \mathrm{g} / \mathrm{m}^{3} \text { valor medio de } 1 \text { hora. } \\
\text { Umbral de alerta: } 240 \mu \mathrm{g} / \mathrm{m}^{3} \text { valor } \\
\text { medio de } 1 \text { hora }\end{array}$ & & $\begin{array}{l}120 \mu \mathrm{g} / \mathrm{m}^{3} \text { (Media octohorania } \\
\text { máxima en um dia). No podrá } \\
\text { superarse más de } 25 \text { dias al año } \\
\text { como promedio de tres años }\end{array}$ \\
\hline Particulas $\mathrm{PM}_{10}$ & $\begin{array}{l}50 \mu g / \mathrm{m}^{3} \text { no podrá superarse en más de } \\
35 \text { veces al año }\end{array}$ & $40 \mu \mathrm{g} / \mathrm{m}^{3}$ & \\
\hline
\end{tabular}

Fuente. Directiva 2008/50/CE y RD 102/2011. 
Los umbrales propuestos por la WHO (World Health Organization) son menos tolerantes, el límite anual del NO2 coincide con el de la legislación europea, pero en las PM10 no debe superar los $20 \mu \mathrm{g} / \mathrm{m}^{3}$ y en el ozono el límite ha descendido a $100 \mu \mathrm{g} / \mathrm{m}^{3}$ de media en 8 horas; además en el último Informe de la WHO (2013) se recomienda endurecer estos valores, todavía más, por los efectos negativos de la contaminación en la salud humana.

\section{DATOS Y METODOLOGÍA}

El ámbito de interés es el municipio de Madrid, pero dada la prioridad sobre los efectos adversos de la contaminación en la población, se ha circunscrito el análisis exclusivamente a la zona urbana poblada, eliminando las partes no urbanas o despobladas. Para ello, a partir de diversas fuentes (Corine Landcover 2006 e imágenes aéreas reicentes) se realizó una identificación de zonas claramente no residenciales, que fueron excluidas del área estricta de estudio. Estas operaciones se apoyaron en la tecnología SIG (ArcGIS). La figura 1 muestra lo que conforma el "área urbana" (AU) de Madrid y las estaciones de la red de vigilancia de la calidad del aire utilizadas.

Los datos utilizados corresponden a la media anual de cada contaminante en el año 2011 de las estaciones de medición de la ciudad de Madrid. Como estas eran escasas se han añadido algunas estaciones de la Comunidad de Madrid, próximas al municipio (figura 1).

Figura 1. Localización espacial de las estaciones de contaminación y área urbana

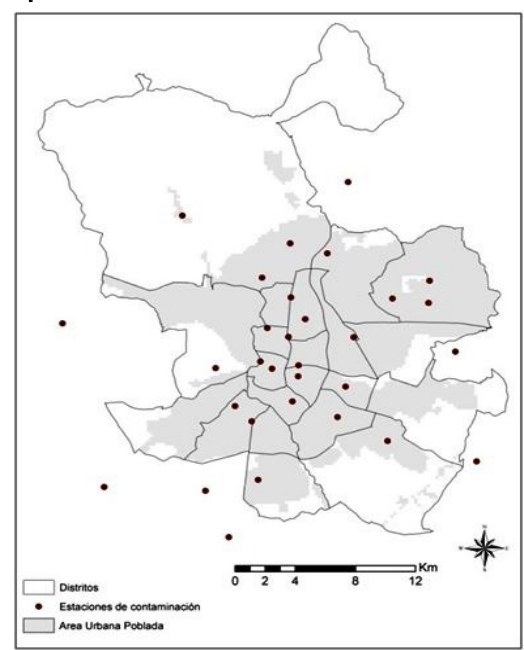


Para disponer de información continua de cada contaminante sobre la totalidad del espacio urbano ( $A U)$, se ha recurrido a la aplicación de técnicas de predicción espacial o técnicas de interpolación, práctica muy habitual en estudios de variables ambientales (Babak y Deutsch, 2009), mediante la utilización de la extensión Geostatistical Analyst de ArcGIS, versión 10.1. El objetivo de la interpolación es detectar el modelo espacial de un fenómeno, estimando valores de zonas donde no hay información a partir de datos muestrales (Diem y Comrie, 2001). El resultado final es un mapa que muestra la distribución espacial del contaminante analizado. Dado que la polución es muy variable, espacial y temporalmente, un análisis más preciso hubiera requerido datos muestrales más abundantes y representativos espacialmente de la contaminación atmosférica, para hacer viable el uso de modelos de difusión / dispersión a escalas grandes, i.e. a nivel de vías y plazas públicas.

Las estimaciones realizadas con la interpolación se han exportado a capas raster con una resolución de $200 \mathrm{~m}$. Con la extensión Spatial Analyst y mediante Raster Calculator se han recortado para ajustarlas a los límites de la zona urbana poblada. En una fase posterior, los datos por píxeles se promediaron para cada distrito lo que ha permitido determinar la calidad ambiental media en los diferentes distritos de la ciudad y estudiar las desigualdades entre ellos.

El método de interpolación finalmente utilizado ha sido el inverso de la distancia (IDW), uno de los que se emplea con mayor frecuencia, por la facilidad de su cálculo y porque no requiere ninguna asunción previa de los datos, pero sobre todo, porque proporciona menores errores en la predicción de los contaminantes. Combina el concepto de proximidad con el de cambio gradual de la superficie de tendencia. Es definido como la media ponderada por el inverso de la distancia dentro de una determinada forma de búsqueda de vecindad. La fórmula para su cálculo es:

$$
Z\left(s_{0}\right)=\sum_{i=1}^{N} \lambda * Z\left(s_{i}\right)
$$

donde ${ }^{Z\left(s_{0}\right)}$ es el valor que se intenta predecir para el lugar $s_{0}, \mathrm{~N}$ es el número de puntos muestrales alrededor del lugar que se va a predecir y que serán tenidos en cuenta en la predicción, $\lambda_{i}$ es el peso asignado a cada punto muestral que se va a usar. Estos pesos decrecen con la distancia y $Z\left(s_{i}\right)$ es el valor observado del lugar $\mathrm{s}_{\mathrm{j}}$. 
La fórmula para determinar los pesos es:

$$
\lambda_{i}=d_{i o}^{-p} / \sum_{i=1}^{N} d_{\infty}^{-p}
$$

A medida que la distancia se hace más grande, el peso es reducido por un factor $p$. Es decir, que según se incrementa la distancia entre los puntos observados y el punto calculado, el peso que tendrá un punto muestral sobre el predicho decrecerá potencialmente; $\mathrm{d}_{\mathrm{i} 0}$ es la distancia entre el lugar de predicción s0 y cada lugar muestral, $\mathrm{s}_{i}$. Los pesos de los lugares utilizados para la predicción serán escalados de tal forma que su suma sea 1 , es decir,

$$
\sum_{i=1}^{N} \lambda_{i}=1
$$

El óptimo valor de p se determina haciendo mínimo el error de predicción o error cuadrático medio (RMSPE en inglés), que tiene la siguiente fórmula:

EMC $=\sqrt{\sum_{i=1}^{n} \frac{\left(\hat{Z}\left(s_{i}\right)-z\left(s_{i}\right)\right)^{2}}{n}}$

El Analista Geoestadístico de ArcGIS, que es la extensión utilizada para realizar estos cálculos, emplea potencias más grandes que 1. Por defecto se usa $p$ $=2$, que se conoce como distancia inversa al cuadrado (Cañada, 2007).

Otra cuestión importante es determinar el número de vecinos a tener en cuenta para calcular el valor predicho. Esto dependerá del tipo de datos y de la superficie que se intente crear. Por defecto el Analista Geoestadístico establece 15 vecinos y un mínimo de 10. Sin embargo los investigadores no se ponen de acuerdo, unos aconsejan entre 3 y 7 vecinos, otros entre 6 y 9 y algunos un número inferior a 6 (Babak y Deutsch, 2009). Al ser el IDW muy sensible al número de vecinos y al valor de $p$ es importante cuidar la elección de estos parámetros.

En el caso de la interpolación de los tres contaminantes del municipio de Madrid, los parámetros aplicados en cada caso, han sido diferentes, siempre buscando aquellos valores que producían un menor error cuadrático medio y una media de los errores más reducida. La forma de búsqueda de vecindad ha sido un círculo, aunque dividido en un número de sectores diferentes pero con idénticas dimensiones del eje, porque el modelo utilizado ha sido el isotrópico (véase tabla 2). Se ha buscado el óptimo valor de $p$. 
Tabla 2. Parámetros utilizados en la interpolación con IDW y resultados de la validación cruzada (crossvalidation)

\begin{tabular}{|c|c|c|c|c|c|c|c|}
\hline Contaminante & $\begin{array}{c}N^{*} \\
\text { Estaciones }\end{array}$ & $\begin{array}{l}N^{\circ} \text { wecinos } \\
\text { Max./Min. }\end{array}$ & $\begin{array}{c}\text { Forma } \\
\text { vecindlad }\end{array}$ & $\begin{array}{c}\text { Dimensiones } \\
\text { Eje en m }\end{array}$ & $\begin{array}{c}\text { Optimo } \\
\text { Valor dep }\end{array}$ & $\begin{array}{l}\text { Modia } \\
\text { emores }\end{array}$ & $R M S P E$ \\
\hline $\mathrm{NO}_{2}$ & 32 & $7 / 3$ & Circulo 8 sectores & 10.343 & 1 & 1,6 & 9,1 \\
\hline $\mathrm{O}_{3}$ & 21 & $10 / 4$ & Circulo 4 sectores & 10.343 & 1,16 & $-0,96$ & 6,2 \\
\hline $\mathrm{PM}_{\mathbf{0}}$ & 19 & $9 / 3$ & Circulo 4 sectores & 10.343 & 1,81 & 0,0 & 3,2 \\
\hline
\end{tabular}

Fuwnte. Elaboración propia.

\section{ANÁLISIS DE RESULTADOS}

El modelo de distribución espacial de los tres contaminantes en el municipio de Madrid es diferente. Si se considera el dióxido de nitrógeno, la mayor parte del espacio incumple la normativa legal vigente; no sucede lo mismo con las $\mathrm{PM}_{10}$ y el ozono. A continuación se examina el patrón espacial estimado de cada contaminante y las diferencias por distritos.

\subsection{El patrón espacial del dióxido de nitrógeno $\left(\mathrm{NO}_{2}\right)$}

La media de los datos estimados por el modelo de interpolación es bastante cerca na a la de los datos observados. En ambos casos el valor medio de la red ha superado el máximo legal $\left(40 \mu \mathrm{g} / \mathrm{m}^{3}\right)$. El valor máximo pronosticado es un poco más bajo y el mínimo un poco más alto. Destaca la menor variabilidad de los valores pronosticados (2,5 frente a 9,3), porque el objetivo de la técnica es hacer mínimo el error de la predicción (tabla 3 ).

El patrón de distribución espacial del $\mathrm{NO}_{2}$ muestra que la mayor parte del municipio rebasa el valor límite anual permitido por la ley $\left(40 \mu \mathrm{g} / \mathrm{m}^{3}\right)$. Los valores más altos se registraron en las zonas de mayor tráfico: eje Castellana-Recoletos hacia el sur y glorieta de Fernández Ladreda. Únicamente algunos enclaves del norte, donde hay nuevos desarrollos urbanos, este y sureste (ensanche de Vallecas) se han mantenido en niveles inferiores al valor límite anual, representados en tonos azules en el mapa (véase figura 2 izqda.). 
Tabla 3. Estadísticos descriptivos de los datos observados y pronosticados de concentración media anual de NO2 en $2011(\mu \mathrm{g} / \mathrm{m} 3)$ en Madrid

\begin{tabular}{|l|c|c|}
\hline ESTADISTICOS & $\begin{array}{c}\text { DATOS } \\
\text { OBSERVADOS }\end{array}$ & PRONOSTICADOS \\
\hline Meclia & 43,2 & 44,63 \\
\hline Des. típica & 9,3 & 2,5 \\
\hline Máximo & 63 & 57,9 \\
\hline Minimo & 23 & 25,03 \\
\hline
\end{tabular}

Fuente. Elaboración propia.

Figura 2. Distribución espacial de la concentración atmosférica de NO2 en 2011 y su distribución por distritos

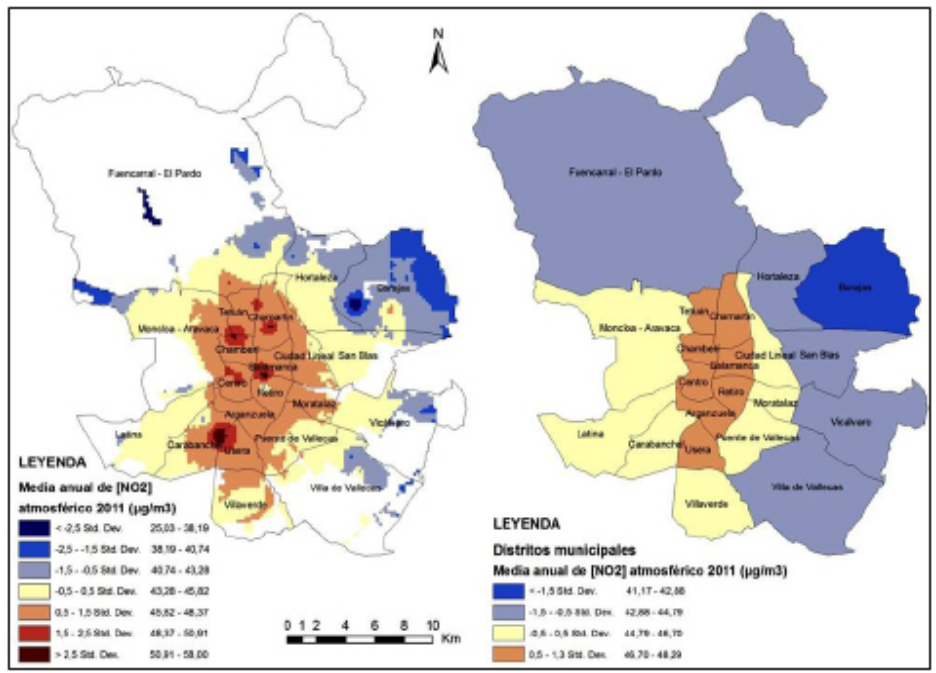

Los factores que explican esta mala calidad del aire, mencionados en otra publicación (Cañada, 2012) tienen que ver, por un lado, por la alta densidad de tráfico rodado en el centro urbano, y entre el centro y la periferia, donde se ubican los nuevos desarrollos urbanísticos, y por otro lado, por el tipo de arquitectura urbana, con vías estrechas, encajonadas; sin olvidar que un clima con frecuentes episodios de calma atmosférica y baja precipitación, como es el de Madrid, no ayuda a la dispersión de la contaminación.

Las desigualdades entre los valores por distritos son pequeñas (coeficientes de variación y de convergencia sigma de 0,04 y 0,05 respectivamente). Se ob- 
serva que los niveles más altos de NO2 se producen en una franja N-S que ocupa el interior y el ensanche y se prolonga hasta la periferia meridional, y el nivel más bajo corresponde a un distrito del E, Barajas. Los niveles medio-bajos engloban a un conjunto de distritos del N, E y SE de Madrid (véase figura 2 dcha.).

La clasificación de los valores por distrito en cuatro intervalos, muestra una clara asimetría negativa y un patrón concéntrico. El mayor número de distritos se concentra en el intervalo superior, con valores por encima de $46,7 \mu \mathrm{g} / \mathrm{m}^{3}$, correspondiendo a ocho distritos del centro y del ensanche. En torno a la media $(45,7 \mu \mathrm{g} /$ $\mathrm{m}^{3}$ ) se congrega otro subconjunto de distritos (en concreto siete) que conforman un arco alrededor de los distritos centrales. El valor más alejado de la media constituye el intervalo inferior, donde se ubica el distrito de Barajas.

El $\mathrm{NO}_{2}$ es un contaminante que afecta a la salud, inhibiendo algunas funciones de los pulmones como la respuesta inmunológica, disminuyendo así la resistencia a infecciones.

\subsection{El patrón espacial del ozono $\left(\mathrm{O}_{3}\right)$}

El ozono es un contaminante secundario que se forma a partir de una serie de reacciones fotoquímicas en las que intervienen la radiación solar, el NO2 y los compuestos orgánicos volátiles (COV) cuando se dan unas condiciones meteorológicas adecuadas, en concreto, en presencia de temperaturas elevadas; esto aclararía por qué los niveles más altos se registran en las tardes de verano. La actividad humana es la principal responsable de los precursores (NO y COV) de este contaminante. La molécula de ozono es muy reactiva y tiende a descomponerse en las zonas en las que existe una alta concentración de monóxido de nitrógeno (NO), lo que explica por qué su presencia es más baja en el centro de las grandes ciudades que en los cinturones metropolitanos o en las áreas rurales circundantes. Por otro lado, el ozono también se ve implicado en fenómenos de transporte atmosférico a grandes distancias por lo que origina problemas de contaminación transfronteriza.

Los estadísticos calculados a partir de datos observados para el conjunto de la red son bastante parecidos a los estimados, sobre todo, la media y el valor mínimo. El valor máximo estimado se ha recortado un poco, pasando de $60 \mu \mathrm{g} / \mathrm{m}^{3}$ a $55,7 \mu \mathrm{g} / \mathrm{m}^{3}$. De nuevo la desviación típica de los valores estimados es más reducida que la de los observados (véase tabla 4). Se recuerda que se ha interpolado el valor medio anual de este contaminante, a pesar de no estar legislado como 
indicador.

Tabla 4. Estadísticos descriptivos de los datos observados y pronosticados de concentración media anual de $\mathrm{O}_{3}$ en $2011\left(\mu \mathrm{g} / \mathrm{m}^{3}\right)$

\begin{tabular}{|l|c|c|}
\hline ESTADISTICOS & $\begin{array}{l}\text { DATOS } \\
\text { OBSERVADOS }\end{array}$ & $\begin{array}{l}\text { DATOS } \\
\text { PRONOSTICADOS }\end{array}$ \\
\hline Media & 45,49 & 45,3 \\
\hline Des. típica & 6,4 & 2,9 \\
\hline Máximo & 60 & 55,7 \\
\hline Mínimo & 32 & 32,6 \\
\hline
\end{tabular}

Fuente. Elaboración propia.

La observación de la figura 3 izquierda permite constatar los siguientes aspectos: la zona con menores valores de ozono, se sitúa en el centro-sur de Madrid, con cifras inferiores a $43,8 \mu \mathrm{g} / \mathrm{m}^{3 ;}$ desde ahí los valores crecen hacia la periferia norte-noreste, donde se alcanzan los $55,7 \mu \mathrm{g} / \mathrm{m}^{3}$. Este modelo es el inverso al mostrado por el $\mathrm{NO}_{2}$, la razón está en las especiales características del $\mathrm{O}_{3}$ como contaminante secundario descritas con anterioridad; son los barrios con menor tránsito de vehículos y con más espacios verdes los que tienen peor calidad del aire según este indicador, ya que en esas zonas escasean las emisiones de óxidos de nitrógeno, que son los que al reaccionar con la molécula de ozono la hacen desaparecer.

Durante 2011 cinco de las catorce estaciones que miden $\mathrm{O}_{3}$ registraron más de 25 superaciones del valor límite legal. Y hubo tres estaciones (Juan Carlos I, Tres Olivos y Barajas Pueblo) que tuvieron superaciones del umbral de información a la población (180 $\mu \mathrm{g} / \mathrm{m} 3$ durante una hora), rebasado el cual, la autoridad competente está obligada a avisar puntualmente a la población, informando sobre las medidas de protección a tomar, especialmente a los colectivos más vulnerables. El modelo de distribución espacial del $\mathrm{O}_{3}$ por distritos muestra una configuración en la que los distritos con niveles más altos ocupan una corona periférica que se extiende por el O, N, E y SE del municipio, mientras que los distritos centrales, del ensanche y del SO exhiben niveles menores (véase figura 3 derecha). El valor medio de todos los distritos ha sido de $45 \mu \mathrm{g} / \mathrm{m}^{3}$ y los coeficientes de variación y de convergencia sigma de 0,06 . Se trata de una situación contrapuesta a la del $\mathrm{NO}_{2}$. 
Figura 3. Distribución espacial de la concentración atmosférica de $\mathrm{O}_{3}$ en 2011 y su distribución por distritos

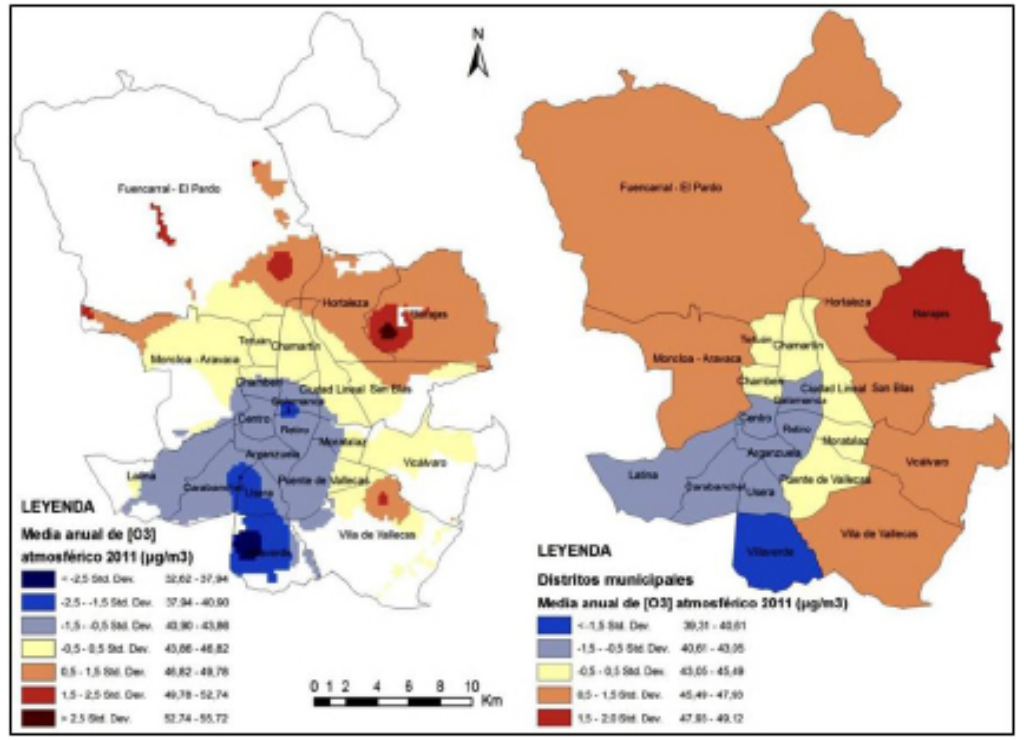

El mayor número de distritos (33\%) se concentran en el intervalo que se sitúa una desviación típica por debajo de la media, con valores que oscilan entre 40,61 y $43,05 \mu \mathrm{g} / \mathrm{m}^{3}$ (corresponden al centro y sur del municipio). En el intervalo de la media, entre 43,05 y $45,49 \mu \mathrm{g} / \mathrm{m}^{3}$, se agrupan el $28,5 \%$ (algunos del centro y ensanche) y el mismo porcentaje se sitúa a una desviación típica por encima de la media (son distritos periféricos del municipio). Los extremos están marcados por Villaverde $\left(39,31 \mu \mathrm{g} / \mathrm{m}^{3}\right)$ de un lado y Barajas $\left(49,12 \mu \mathrm{g} / \mathrm{m}^{3}\right)$ del otro (véase figura 3 derecha).

El exceso de ozono en el aire puede producir efectos adversos de consideración en la salud humana. Puede causar problemas respiratorios, provocar asma, reducir la función pulmonar y originar todo un cuadro de enfermedades pulmonares. Actualmente se trata de uno de los contaminantes atmosféricos que más preocupan en Europa. Diversos estudios europeos han revelado que la mortalidad diaria y mortalidad por cardiopatías se incrementan un $0,3 \%$ y un $0,4 \%$ respectivamente con un aumento de $10 \mu \mathrm{g} / \mathrm{m}^{3}$ en la concentración de ozono. La respuesta a la exposición de este contaminante puede variar mucho entre individuos por razones genéticas, edad y por la presencia de afecciones respiratorias como alergias y asma, cuyos síntomas son exacerbados por el ozono. El impacto de este tipo de polución es elevado en la calidad de vida y bienestar de los ciudadanos, y concierne también a la sostenibilidad y la justicia ambiental. 


\subsection{El patrón espacial de las $\mathrm{PM}_{10}$}

Las partículas en suspensión (aerosoles) son elementos sólidos o líquidos dispersos en el aire de origen natural (partículas de arena o tierra levantada por el viento, incendios forestales, erupciones volcánicas, sal marina) o artificial (partículas de combustión tanto industrial como urbana o residencial, emisiones industriales, tráfico, minería, cementeras, humo de tabaco, empleo de aerosoles, etc.). No se trata de un contaminante único y homogéneo, sino de un conjunto de contaminantes de muy diversa naturaleza, tanto en su tamaño como en su composición. Las partículas sólidas suelen ser de origen mineral (sílice) u orgánico. En ambientes urbanos, donde la principal fuente de emisión es el tráfico, tienen mucha importancia las partículas de carbono procedentes de los humos de los motores diésel, debidas a una combustión incompleta que, además, tienen mucha capacidad para ensuciar y decolorar las superficies de los diversos materiales de los edificios. Asimismo, también se trata de partículas de origen animal o vegetal, bacterias, hongos, polen, etc. Las partículas líquidas están constituidas por pequeñas gotitas de agua. Desde el punto de vista medioambiental se consideran dos fracciones de partículas en suspensión diferenciadas por tamaño que, por otra parte, tienen diferentes orígenes y efectos sobre la salud de las personas expuestas: son las fracciones de partículas menores de 10 micras $\left(\mathrm{PM}_{10}\right)$ y de 2,5 micras $\left(\mathrm{PM}_{2^{\prime}, 5}\right)$. En nuestros estudios se utiliza el primer indicador, por disponer de una red de medición más amplia.

La legislación vigente establece dos tipos de valor límite de contaminación por PM10 para la protección de la salud humana: un valor límite anual y un valor límite diario. En cuanto al primero de ellos, no debe exceder los $40 \mu \mathrm{g} / \mathrm{m3}$, sin embargo la Organización Mundial de la Salud recomienda no superar los $20 \mu \mathrm{g} / \mathrm{m}^{3}$ para una adecuada protección de la salud humana.

El valor límite diario de $\mathrm{PM}_{10}$ está fijado en $50 \mu \mathrm{g} / \mathrm{m}^{3}$, que según la legislación actual no debe superarse más de 35 días al año. En 2011 ninguna estación registró superaciones, si se descuentan en el cómputo anual las contribuciones de fuentes naturales, tal como prevé la legislación.

Los valores de $\mathrm{PM}_{10}$ del año 2011 indican que todas las estaciones están lejos del límite establecido por la normativa de la UE. Sin embargo, 11 de las 12 estaciones que miden $\mathrm{PM}_{10}$ superaron el valor límite anual recomendado por la OMS, así como el valor medio de la Red, que alcanzó los $24,21 \mu \mathrm{g} / \mathrm{m}^{3}$. La proximi- 
dad entre los estadísticos descriptivos de los valores pronosticados respecto a los observados es una prueba de la fiabilidad del ajuste realizado (tabla $n^{0} 5$ ).

Tabla 5. Estadísticos descriptivos de los datos observados y pronosticados de concentración media anual de $\mathrm{PM}_{10}$ en $2011\left(\mu \mathrm{g} / \mathrm{m}^{3}\right)$

\begin{tabular}{|l|c|c|} 
ESTADISTICOS & $\begin{array}{l}\text { DATOS } \\
\text { OBSERVADOS }\end{array}$ & $\begin{array}{l}\text { DATOS } \\
\text { PRONOSTICADOS }\end{array}$ \\
\hline Media & 24,21 & 23,8 \\
\hline Des. típica & 3,6 & 1,7 \\
\hline Máximo & 30 & 29,9 \\
\hline Minimo & 19 & 19 \\
\hline
\end{tabular}

Fuente. Elaboración propia.

El examen de la distribución espacial estimada de este contaminante pone de manifiesto dos zonas donde se dan las mayores concentraciones de PM10; una corresponde a barrios del centro y sur de la ciudad y otra al extremo este-sureste, en torno a Vicálvaro y Vallecas (figura 4 izquierda). Las razones que explican esta disposición son, por un lado la alta densidad de tráfico de algunos de esos barrios, alrededor del Paseo de la Castellana, Glorieta de Gregorio Marañón y Cuatro Caminos; y por otro lado, además del tráfico (Glorieta de Fernández Ladreda), hay que mencionar que en otros casos son ámbitos con una pobre cobertura vegetal, con grandes espacios de suelos secos (Villa de Vallecas y Vicálvaro), más fácilmente erosionables y expuestos todo el año a la acción del viento que levanta gran cantidad de polvo en suspensión.

El valor medio de este indicador por distritos en 2011 es de $24,4 \mu \mathrm{g} / \mathrm{m}^{3}$. Sus coeficientes de variación $(0,05)$ y de convergencia sigma $(0,08)$ son muy reducidos lo que indica una poca variabilidad entre distritos.

La agrupación de los datos en cuatro clases (figura 5 dcha.) refleja la coincidencia de la moda y de la media en el mismo intervalo $\left(23,8-25,05 \mu \mathrm{g} / \mathrm{m}^{3}\right)$ donde se concentran el $38 \%$ de los distritos. El 33\% de los mismos está entre la media más una desviación típica $\left(25,05-26,32 \mu \mathrm{g} / \mathrm{m}^{3}\right)$. El tramo más bajo está representado por Fuencarral-El Pardo y Hortaleza. La distribución presenta una ligera asimetría negativa. 
El mapa por distritos muestra una persistencia del patrón espacial de este indicador en la ciudad (vid. figura 4 dcha.). Se observan dos zonas más desfavorecidas con superior concentración de partículas, una corresponde a los distritos de la zona centro (Chamberí, Salamanca, Centro y Retiro) y la otra a distritos del S y SE (Villaverde, Villa de Vallecas y Vicálvaro). Los distritos con menor concentración de partículas, que corresponden a zonas con menos tráfico y más espacios verdes, se localizan en el O-NO (Moncloa-Aravaca y Fuencarral-El Pardo), N y E (Chamartín, Hortaleza, Barajas).

Este indicador incide sobre el bienestar y la calidad de vida porque la alta concentración de PM10 en la atmósfera afecta a la salud ciudadana, incrementando la mortalidad por enfermedades respiratorias y cardiovasculares, e incluso puede reducir la esperanza de vida. También concierne a la justicia ambiental porque se ha apreciado que algunos distritos del $\mathrm{S}$ con niveles altos de partículas son los que poseen además rentas más bajas (Cañada et al., 2011).

Figura 4. Distribución espacial de la concentración atmosférica de PM10 en 2011 y su distribución por distritos

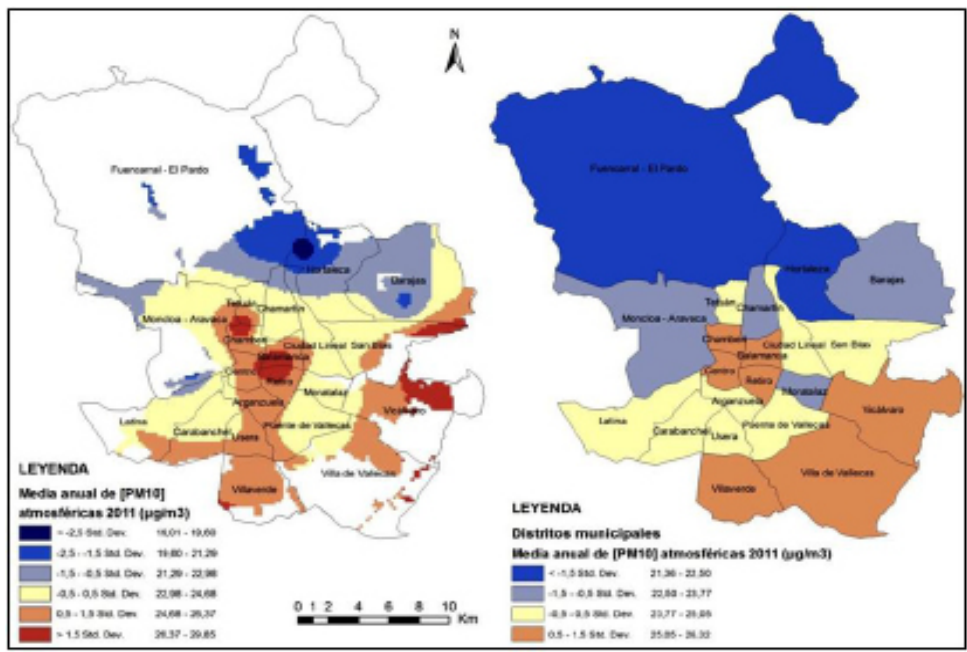

\section{BALANCE Y CONCLUSIONES}

El seguimiento de los logros de las políticas urbanas está requiriendo de evaluaciones ex post con un alto contenido geográfico. A tal fin, las administraciones territoriales se están sensibilizando del valor que aporta ese conocimiento, 
cuya generación precisa, en muchos casos, recurrir a técnicas y geotecnologías de cierta sofisticación, pero cuya potencia posibilita abordar cuestiones complejas, tales como la de dirimir si las estructuras urbanas avanzan hacia escenarios más o menos equilibrados.

Como ilustración del conocimiento producido con geotecnologías (SIG) para tales fines se han mostrado aquí algunos análisis que regularmente se ejecutan para evaluar las desigualdades dentro del municipio de Madrid, mediante el concurso de técnicas de interpolación, que permiten generar una superficie continua de información ambiental con la que caracterizar las distintas zonas de la ciudad.

Metodológicamente se ha empleado la técnica del Inverso de la Distancia (IDW) con modelo isotrópico, aplicando distintos parámetros según cada contaminante. En próximos trabajos se utilizará el modelo anisotrópico con variaciones en sus parámetros, que está consiguiendo mejorar un poco la bondad de ajuste de los resultados.

El patrón espacial de cada contaminante resulta diferente. La calidad del aire en cuanto al $\mathrm{NO}_{2}$ es mala en casi toda la superficie urbana, salvo pequeños enclaves periféricos por el oeste, norte y noreste del Municipio; son los distritos con mayor tráfico los que presentan peor situación. Un patrón contrapuesto al anterior exhibe el $\mathrm{O}_{3}$, son las zonas con menor densidad de tráfico y más espacios verdes las que soportan tasas más elevadas de contaminación por ozono. En cuanto al análisis de las $\mathrm{PM}_{10}$ vuelven a ser los distritos del centro, sur y este los que más sufren las consecuencias de este contaminante, que por otra parte incumplen la normativa marcada por la OMS.

Los parámetros utilizados para ver los desequilibrios entre distritos indican que las diferencias entre ellos, aunque existen, son pequeñas. $\mathrm{El} \mathrm{NO}_{2}$ y las $\mathrm{PM}_{10}$ presentan contrastes similares, las diferencias aumentan si se tiene en cuenta al ozono.

En resumen, procede insistir en que este tipo de análisis tiene como finalidad principal ayudar a los políticos y otros agentes sociales a valorar los planes y programas que inciden sobre el medio ambiente urbano, los cuales deben avistar la mejora de la calidad del aire, paliando los desequilibrios e inequidades socio-ambientales entre barrios. 


\section{BIBLIOGRAFÍA}

- Cuadrado Roura, J. R., Marcos Calvo, M. A. y Lisbona Fuentes, L. (2005). Reequilibrio territorial. Estudio comparativo. Situación social y económica en la ciudad de Madrid. Madrid, Ayuntamiento de Madrid, Observatorio Económico.

- Babak, O. y Deutsch, C.V. (2009): "Statistical approach to inverse distance interpolation", Stoch. Environ. Res. and Risk Assess, 23, págs.543-553.

- Cañada, R. (2007): "Técnicas de interpolación espacial deterministas y exactas: media ponderada por el inverso de la distancia y funciones de base radial", en Moreno, A. (Coord.): Sistemas y análisis de la información geográfica. Manual de Autoaprendizaje con ArcGIS. Ra-Ma, págs. 791-819.

- Cañada, R., Vidal, M. J. y Moreno, A. (2011): “Interpolación espacial y visualización cartográfica para el análisis de la justicia ambiental: ensayo metodológico sobre la contaminación por partículas atmosféricas en Madrid", GeoFocus (Artículos), 11, págs. 118-154. http://geofocus.rediris.es/2011/Articulo6_2011.pdf

- Cañada, R. (2012): Estimación y Diagnóstico Espacio-Temporal de la Contaminación por Dióxido de Nitrógeno en el Municipio de Madrid (2001-2011). Comunicación al XV Congreso Nacional de Tecnologías de la Información Geográfica, Madrid, CHSS-CSIC, págs.. 265-277. ISBN: 978-84-695-4759-5.

- Barómetro de Economía Urbana (2007): "Equilibrio territorial: Situación y evolución del equilibrio territorial en la Ciudad de Madrid", Barómetro de Economía Urbana, 12, abril 2007, Ayuntamiento de Madrid. http://www.madrid.es/ UnidadesDescentralizadas/UDCObservEconomico/BarometroEconomia/2007/ Ficheros/Abril/Equilibrio\%20territorial.pdf

- De Mesnard, L. (2013): "Pollution models and inverse distance weighting: some critical remarks", Computers and Geosciences, Vol. 52, págs. 459-469.

- Diem, J.E. y Comrie, A.C. (2002): "Predictive mapping of air pollution involving sparse spatial observations", Environmental Pollution, 119, págs. 99-117.

- Moreno Jiménez, A. y Vinuesa Angulo, J. (2009): "Desequilibrios y reequilibrios intrametropolitanos: principios de evaluación y metodología de análisis", Ciu- 
dad y Territorio - Estudios Territoriales, LXI, 160, págs. 233-262.

- Moreno, A., Vinuesa, J. y Cañada, R. (2011): Los desequilibrios y reequilibrios intraurbanos en Madrid: diagnóstico 2011, Barómetro de Economía de la Ciudad de Madrid, 30, octubre, págs. 133-168.http://www.esmadrid.com/recursos/ doc/es/Negocio/ObservatorioEconomico/1182573588_62201213348.pdf

- Moreno, A., Vinuesa, J. y Cañada, R. (2012): Los desequilibrios y reequilibrios intraurbanos en Madrid: diagnóstico 2012, Barómetro de Economía de la Ciudad de Madrid, 34, octubre, págs. 81-120. http://www.esmadrid.com/recursos/ doc/es/Negocio/ObservatorioEconomico/1199732380_28122012143144.pdf

- WHO (2013). REVIHAAP (Review of evidence on health aspects of air pollution). World Health Organization, Regional Office for Europe, págs. 33. http:// www.euro.who.int/_data/assets/pdf_file/0020/182432/e96762-final.pdf 